schiede von demjenigen, den wir soeben kennen gelernt haben. Die Steigerung der N-Ausscheidung ist nehmlich an Tage der künstlichen Erwärmung selbst nur sebr wenig ausgesprochen, erreicht am nachfolgenden Tage ihr Maximum, und sinkt dann rasch ab, so dass sie am dritten Tage nach der Ueberhitzung sogar etwas unter der Norm ist.

A us diesem wesentlich verschiedenen Veriaufe der vermehrten N-Ausscheidung in den Versuchen von Richter und in den meinigen wird man scbliessen dürfen, dass die durch Tetrahydro- $\beta$-naphtylamin bedingte Vermehrung des Eiweisszerfalls nicht ausschliesslich Folge der durch das Gift hervorgerufenen T'emperatursteigerung ist, sondern zum Theil auch eine specifische Wirkung des Giftes darstellt.

\title{
3.
}

\section{Ueber die Beziehungen des Carcinoms zur Pachydermie.}

Aus dem pathologisch-anatomischen Institut zu Berlin.

\author{
Von Dr. A. Kuttner, \\ Assistentén an der Poliklinik von Prof. H. Krause in Berlin.
}

In No. 25 der deutschen medic. Wochenschrift (XVI. Jahrg.) findet sich ein Artikel von Hern Prof. E. Klebs, betitelt: Carcinow oder Pachydermie? Die in demselben auf Grund der mikroskopischer Untersuchung von zwei Fillen niedergelegten Ansichten unterscheiden sich in wesentlichen Punkten von den Principien der Virchow'schen Schule, zu denen auch ich wich nach einer grösseren Reihe diesbezüglicher Untersuchungen voll und ganz bekenne, so dass mir zur Klärung der Sache eine Betonung des beiderseitigen Standpunktes wünschenswerth erscheint.

Der erste von Klebs bier angezogene Fall dürfte dem Kliniker allerdings sind die klinischen Daten nur skizzenbaft wiedergegeben - nicht gerade als eiu typischer Fall von Carcinoma largngis erscheinen: doch die Entscheidung der Diagnose liegt ja, wenn der pathologische Anatom eine bejahende Antwort zu geben vermag, rückbaltslos bei diesem. Noch aber besteht das Postulat, das Virchow für die Diagnose des Krebses als unerlässlich hingestellt hat, unerschüttert und zu Rectit fort: Das Vorkommen epithelialer Gebilde im Untergewebe ist das Entscheidende; überall. da, wo am Grunde der Epitheldecke die Grenze zwischen dieser und dem Bindegewebe eine normale, scharfe ist, bandelt es sich un rein locale Prozesse; nur da, wo unterhalb dieser Grenze sich mit der Oberfläche nicht zusammenhängende (eine entsprechende Schnittrichtung natürlich vorausgesetzt) epitheliale Gebilde vorfinden, ist der Beweis für die Malignität des betreffenden Tumors erbracht.

Ws findet sich nun in der ganzen Besprechung dieses Falles nur eine einzige Stelle, welche dieser Forderung genügen dürfte. S, 540 sagt Klebs: 
„In Schnitt 5 aber findet sich eine grosse längliche Epithelzelle, der Innenfiäche eines perivasculären Lywphraumes angelagert.". Wenn ich auch weiss, wie schwer es ist, in einem perivasculären Lymphranm mit absoluter Sicherheit eine Zelle als Epithelzelle zu erkennen, so würde ich mir doch an den thatsächlichen Befunden des hochverdienten Forschers nie einen Zweifel erlauben: nur über die Detutung und Verwerthung dessen, was wir gesehen, herrscht Uneinigkeit. Diese eine Zelle, - ich nehme an, dass sie in keinem continuirlichen Zusammenhang mit der Epitheldecke stand, - beweist nach Virchow's Forderung das Carcinom; denn Vilchow sagt: „Hier (d. h. im Untergewebe) darf absolut nichts mehr existiren, was auch nur entfernt den Eindruck eines Epithelialgewebes macht. Jede Spur von Epithel im Bindegewebe halte ich für suspect."

Alles andere aber, was Klebs von diesem Falle berichtet, ebenso wie die auf $\mathrm{S} .538$ beigefügte $\mathrm{Ab}$ bildung kann ich für in keiner Weise charakteristisch für Carcinom balten.

Die Abbildung zeigt das Bild einer Pachydermie, wie sie durch die verschiedensten Anlässe bedingt sein kann: Verhornung der obersten Epithelschichten; Epithelzapfen, die zum Theil Jängs, zum Theil quer getroffen sind; hierzu die üblichen Veränderungen im Bindegewebe und die Erweiterung einzelner Blutgefässe, wie man sie bald schwächer, bald stärker ausgebildet bei allen möglichen chronischen Entzündungsprozessen antrifft. Es ist wohl möglich, dass dieses Bild aus der Umgegend eines Carcinoms stammt; aber die Diagnose auf Krebs lässt sich aus ihm entschieden. nicht stellen.

Dér Erweiterung der Blutgefässe, welche nach Klebs oft so hochgradig ist, dass sich formliche Blutcysten bilden, und der er eine gani besondere Wichtigkeit für die Krebsdiagnose vindicirt, konnte ich eine so charakteristische Bedeutung bisher nicht zuerkennen. Ich habe eine grosse Anzahl von mikroskopischen Präparaten, die unzweifelhaften Kehlkopfcarcinomen entstammten (in den lefzten 7-8 Monaten allein haben wir 6 Mal die klinische Diagnose des Carcinoma laryngis aus der Praxis und Poliklinik meines hochverehrten (hefs, des Herrn Prof. H. Krause, durch die wikroskopische Untersuchung zu bestätigen Gelegenheit gehabt), mit Rücksicht auf diese Verbältnisse von neuem durchgesehen; oft fand ich Blutgefässe, die unmittelbar an den Epithelzapfen anlagen, ohne dass sie irgendwie dilatirt oder in ihren Wandungen rareficirt waren; oft auch begegnete ich den angegebenen Veränderungen, fand sie aber auch, and das ist wohl das Entscheidende, bei den verschiedensten Zuständen von Pachydermie, die rein local waren und local bfieben. So liegt vor mir ein Präparat, herrührend von einer syphilitischen Pachydermie, das eine überaus grosse Aehnlichkeit wit der Klebs'schen Abbildung zeigt: ein mächtiges Blutgefäss liegt dem tiefsten Theile eines Epithelzapfens so innig an, dass derselbe, durch den Widerstand des Gefässes wahrscheinlich in seiner Wachsthumsrichtung beeinflusst, sich in leicht S-förmiger Krümmung an dasselbe anschmiegt. Trotz dieser innigen Beziebung zwischen Epithelzapfen und Blutgetäss war aber cer Prozess, wie 
schon angedeutet, dauernd ein rein localer, den jeder chirurgische Eingriff nur ungünstig hätte beeinflussen bönnen.

Die Eanntbedeutung der Klebs'schen Arbeit würde aber in den praktischen Schlussfolgerungen liegen, die der Verf. aus seinen Beobachtungen ziehen zu müssen glaubt. Er schreibt: „Lie pachyderme Bildung leitet ganz allmählich zur Carcinombildung hinüber, und dieser Uebergang findet durch ein alltnähliches Einwuchern der hyperplasirenden Epithelien in die Lymphund Blutgefässäume statt." Es will mir scheinen, als ob dieser Erklärungsversuch, der sich auf rein mechanische Verhältnisse stützt, die Differenzen, die in dem Wesen des Carcinoms und der Pachydermie begründet liegen; nicht genügend berücksichtigt. Charalsteristisch für das Carcinom ist die ausserordentlich vitale Energie seiner Elemente, der kein Gewebe auf die Dauer widerstehen bann, - eine Energie, die alles, was sich ihr in den Weg stellt, ausnahmslos vernichtet. Charakteristisch ist forner für das Carcinom, die verderbliche Fähigkeit der Generalisation durch den ganzen Organismus, wenn auch gerade dieser Punkt beim Larynxcarcinom durch die hier herrschenden eigenthümlichen anatomischen Verhältnisse mehr in den Hintergrund tritt. Im directen Gegensatz hierzu steht die Pachydermie, welcher Art sie auch immer sei: sie ist und bleibt immer loca); sie generalisirt sich nie und vermag nie activ ein Gewebe zu vernichten. Sollte nun das Hineinwachsen einer einzigen Epithelzelle in ein Lymph- oder Blutgefäss genügen, um diesen durchaus localen Prozess von Grund aus in seiner Natur und seinem Wesen umzuwandeln? Was beweist uns, dass durch diesen rein mechanischen Vorgang die vitale Energie aller Elemente plötzlich zum Verderben des ganzen Organismus bis in's Unendliche gesteigert werde? Was beweist auch nur, dass diese im Gefässlumen liegende Zelle thatsächlich die Kraft besitze, losgerissen vom Nutterboden nach ibrer Verschleppung an einen anderen Ort hier den ersten Keim zur Bildung einer neuen Geschwulstmasse abrugeben?

Und noch ein anderer Grund spricht, scheint wir, gegen diese Theorie. Kanthack, voll und ganz auf dem Boden der Virchow'schen Deduction stehend, hat des weiteren ausgeführt, dass eine Pachydermie veranlasst werden könne durch chronische Prozesse rein entzündlicher Natur (Pachyd. simplex), ebenso gut wie durch Tuberculose und Lues ( $P$. tuberculosa et syphilitica). Sollte es nun wahrscheinlich sein, dass Tuberculose oder Syphilis im Kehlkopf auf dem Umwege einer Pachydermie zum Carcinom führt? Und das müsste doch nach den Klebs'schen Anschauungen oft genug dadurch geschehen, dass eine Epithelzelle aus solch' einer Pachydermie in das Innere eines Blut- oder Lymphgefässes geriethe!

Nicht unzweideutig erscheint auch der zweite von Klebs angeführte Fall. Einmal müssen wohl Larynxcarcinome, welche in Gestalt eines gestielten Polypen herunterhängen, in denen nur der Polyp, nicht aber die $B$ asis carcinomatös ist, enorm selten sein; denn bei v. Zjemssen, Fauvel, Mackenzie, Butlin, Störk, Türk, Gottstein, B. Fränkel babe ich vergeblich nach ähnlichen Fällen gesucht, habe auch niemals Gelegenheit 
gebabt einen derartigen Fall selbst zu beobachten. Es mag woll yolkommen, dass ein knotenförmiges Carcinom sich allmählich mehr und mebr hervordrängt und schliesslich Polypenform annimmt, dann war doch aber immer erst die Basis erkrankt; dass aber ein Larynxpolyp sich als carcinomatös degenerirt erweist, während die Basis - und das scheint mir aus der Krankengeschichte hervorzugehen - vollständig normal ist, ein derartiger Fall wäre ein Unicum!

Untersucht man eine grössere Anzahl von Stimmbandpolypen (meistentheils handelt es sich um cystenreiche Fibrome), so findet man, dass der mehr oder weniger kugelförmige fibröse Grundstock von einer mächtigen Hülle von Plattenepithelien umgeben jst, die in ihren obersten Schichten. meistentheils verbornen, während nach unten hin in das fibröse Gewebe nicht selten mächtige Epithelzapfen eindringen, die, wenn auch in Zickzacklinien, ungefähr doch die Richtung nach der Mitte des Tumors hin nehmen. Schneidet man nun solch' einen Polypen, - und von einer richtigen Schnittrichtung kann man doch bei einem annähernd kugligen Gebilde nur in sehr beschränktem Sinne sprechen, - so sieht man öfters unter dem Mikroskop mitten im Bindegewebe isolirt erscheinende Zapfen, die leicht den Eindruck erwecken können, dass es sich um einen malignen Tumor handele. Deshalb darf man in einem solchen, immerbin schwierig zu deutenden Falle die Diagnose auf Krebs erst dann für gesichert erachten, wenn auch die Basis des Polypen sich.als carcinomatös herausstellt. Da die Basis aber gar nicht untersucht worden ist und Klebs ausdrücklich sehreibt: „Die am 18. Tage nach der Operation (Abhebung des Polypen mit der Schrötter'schen Pincette) vorgenommene Unter'suchung ergab einen ganz glatten Stimmbandsanm; die Dicke desselben normal; keine Infiltration am Stimmband erkennbar. Pat. singt mit Bruststimme vom tiefen a bis zum fis der obersten Notenlinie. Auch am 106. Tage nach der Operation konnte das gleiche Verhalten bestätigt werden," so glaube ich, dass sowohl klinisch wie pathologisehanatomisch die Deutung dieses Falles bis auf Weiteres in suspenso bleiben muss. -

So dankenswerth auch die Bemübungen unseres Autors sind, so wichtig es auch gerade für den Kliniker wäre, wenn wir Mittel und Wege an die Hand bekämen, um das Careinom in seinen ersten Ạ̧fängen unizweifelhaft zu erkennen, so erscheint es mir nach alledem doch mehr als zweif elhaft, ob die von Klebs angegebenen Kriterien sich zu diesem Zweck verwerthen lassen. Die Erweiterung und innige Anlagerung von Blutgefässen an Epithelzapfen, auf welche die Klebs'schen Auseinandersetzungen sich basiren, sind, wie wir nachgewiesen zu haben glauben, nicht charakteristisch für Carcinom: man findet beim Keblkopfkrebs die beschriebenen Verhältnisse, man vermisst sie auch oft, - man findet sie aber, und das ist der wesentlichste Punkt, der die Klebs'sche Theorie zu Falle bringt, auch bei den verschiedenartigsten Pachydermien, also bei Prozessen, die rein localer Natur waren und blieben. Und mit der Prämisse fallen anch die aus ihr gezogenen therapeutischen Schlussfolgerungen. 


\section{2}

Warnen muss man davor, und ich glaube mich bier der Zustimmung aller Laryngologen versichert halten zu dürfen, überall da zu operiren, wo "neben den Zellzapfen sehr weite, klaffende Blutgefässe vorbanden sind, an deren Oberff̈che sich die Zapfen dicht anlagern". Selbst wenn wir, was Klebs im Uebrigen gar nicht einmal ausdrücklich perlangt, auf's Sorgsamste alle syphilitischen und tuberculösen Pachydermien auszuschliessen versuchten, so weit das eben praktisch angebt, wir würden, wenn wir den KJebs'seben Grundsätzen folgten, noch viel zu häufig operiren.

Liegt. erst einmal ein Carcinoma laryngis vor, so kann man ron einem geringfügigen endolaryngealen. Kingriff kaum mehr einen Frfolg erwarten: eine Laryngotomia externa aber mit einer theilweisen oder gar vollständigen Ausräumung des Kehlliopfes ist doch eine so eingreifende Operation, dass sie nur durch die allerzwingendsten Gründe gerechtfertigt werden kann.

Nach R. Virchoin's lichtvollew Vortrage, der nachher durch die Kanthack'schen Arbeiten noch in dankenswerther Weise ausgebaut warden ist, liegen alle Verhältnisse dieser heut nicht mehr "neuen" Erkrankung, die überdies so viele Analoga in anderen Körperregionen aufweist, dass man sie kaum „eine eigenartige" nennen kann, klar.

Uebergiebt der Kliniker dem pathologischen Anatomen ein Gewebsstück, das in genügender Grösse a us den Tumor selbst entfent ist, so macht die Diagnose keinerlei Schwierigkeit.' Ist das excidirte Stück aber nur aus der Oberfläche entfernt, so dass es nichts vom Untergewebe enthält oder stammt es gar nur aus der Umgebung des Tumors, nicht aus diesem. selbst, so bleibt der patbologische Anatom frei von jeder Verautwortung.

Bis auf Weiteres müssen wir daher an den von $V$ irchow klar formulirten Postulaten festbalten; nur wenn diese erfült sind, sind wir berechigt, die mikrostopische Diagnose auf Carcinom zu ștellen, $\bar{x}$ und nur erst, wenn die Diagnose über allen $Z$ weifel erbaben ist, darf meines Erachtens der Kiinilzer zum Messer greifen! - 